\title{
Coagulative Necrosis
}

National Cancer Institute

\section{Source}

National Cancer Institute. Coagulative Necrosis. NCI Thesaurus. Code C39608.

Ischemic tissue necrosis characterized by the preservation of the architectural pattern and a necrotic ghost appearance of the cells. 